\title{
Gastroduodenal artery pseudoaneurysm resulting from a plastic stent after pseudocyst drainage
}

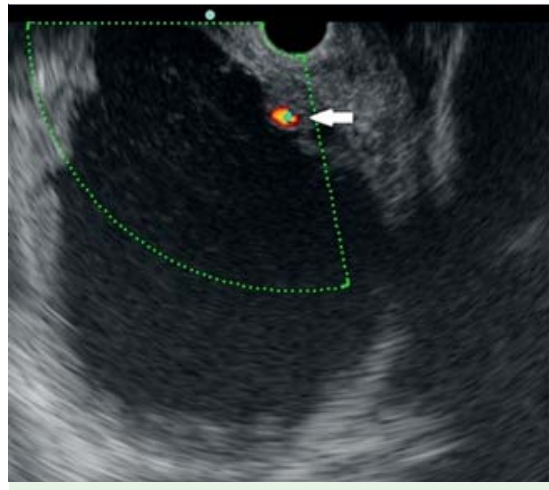

Fig. 1 In a 22-year-old man undergoing transmural drainage of a pancreatic pseudocyst and placement of a 7-Fr, 5-cm doublepigtail plastic stent, endoscopic ultrasound shows the pseudocyst with the gastroduodenal artery coursing in its wall (arrow).

A 22-year old man with posttraumatic pancreatitis had a $6-\mathrm{cm}$ pseudocyst in the head and body of the pancreas and pancreatic ascites. When he presented with abdominal pain, endoscopic ultrasound (EUS)-guided transmural drainage of the pancreatic pseudocyst was performed, and a 7-Fr, 5-cm double-pigtail plastic stent was placed in the cavity. During EUS, the gastroduodenal artery (GDA) was seen coursing through the cavity of the pancreatic pseudocyst ( $\bullet$ Fig. 1 ), but neither EUS nor contrast-enhanced computed tomography identified a pseudoaneurysm. Endoscopic retrograde pancreatography (ERP) revealed complete disruption of the pancreatic duct. After a marked improvement in the patient's symptoms, he was discharged.

However, 4 weeks later, the patient presented with hematemesis associated with abdominal pain, melena, postural symptoms, tachycardia, and hypotension. Investigations revealed a hemoglobin level of $7.6 \mathrm{~g} / \mathrm{dL}$. He was resuscitated and given 3 units of blood. Urgent gastroscopy showed blood oozing through and adjacent to the transmural stent. Computed tomographic angiography of the abdominal vessels did not reveal any vascular abnormality, but the GDA was seen closely abutting the transmural stent ( $\bullet$ Fig. 2 ). The pseudocyst had resolved ( $\bullet$ Fig.3), but ascites was present.

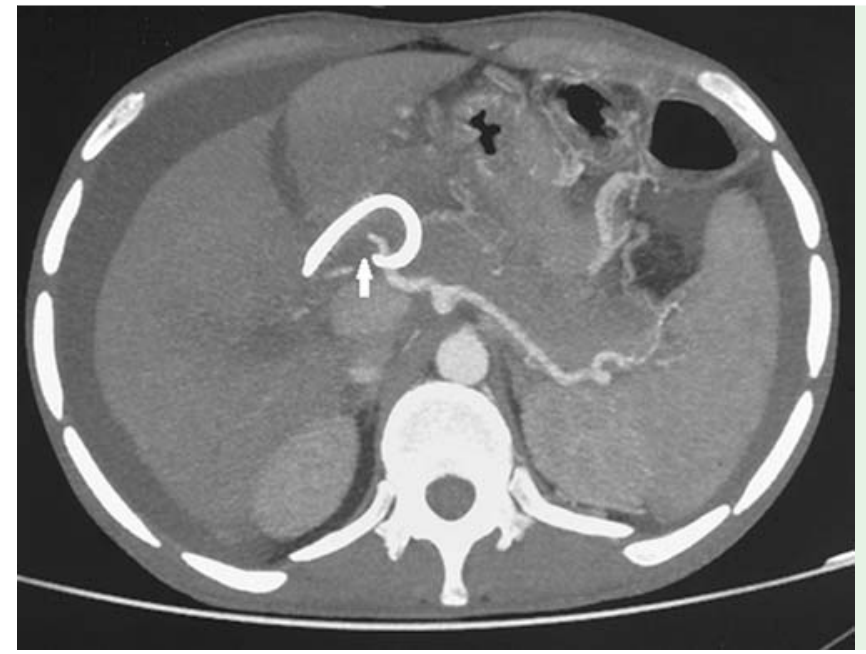

Fig. 2 Computed tomographic angio graphy shows the gastroduodenal artery coursing close to the tip of the stent (arrow).

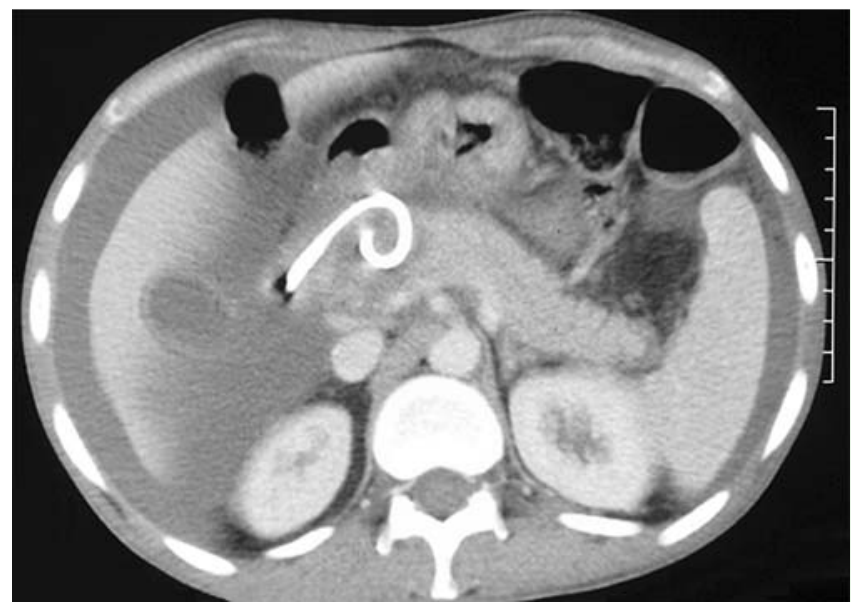

Fig. 3 Computed tomography of the abdomen shows the collapsed pseudocyst with the stent in situ.

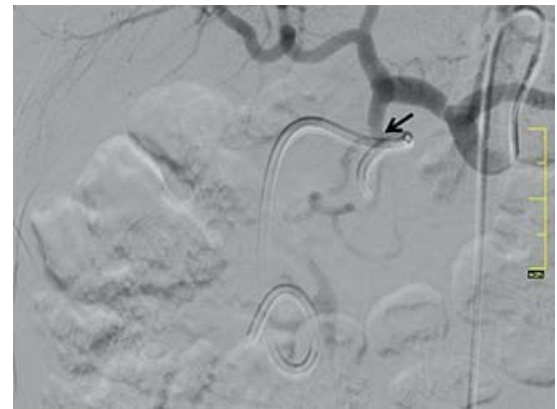

Fig. 4 Digital subtraction angiography of the celiac artery shows luminal irregularity of the segment of the gastroduodenal artery abutting the stent (arrow).

In view of the ongoing bleed, digital subtraction angiography (DSA) was done and showed luminal irregularity and attenuation of the segment of the GDA that was abutting the stent ( $\bullet$ Fig. 4 ). The GDA was

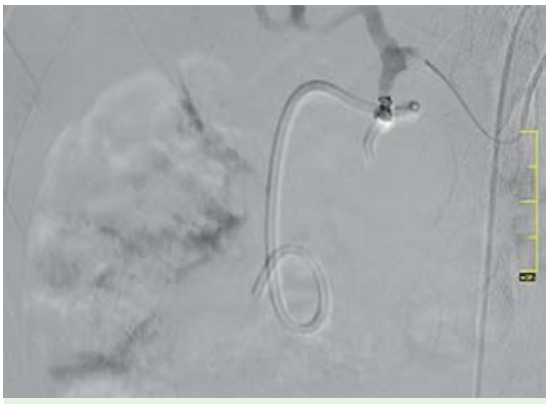

Fig.5 Digital subtraction angiography of the common hepatic artery following coil embolization reveals non-opacification of the affected segment of the gastroduodenal artery.

embolized with coils ( $\bullet$ Fig.5), after which the bleeding stopped. Because the patient still had ascites and a disrupted pancreatic duct, the transmural stent was replaced with a shorter $7-\mathrm{Fr}, 3-\mathrm{cm}$ stent. 
Following this, the ascites resolved, and the bleeding did not recur over a followup period of 6 months.

GDA pseudoaneurysms are commonly caused by pancreatitis, vascular interventions, trauma, or surgery [1]. They result from a breach in the vessel wall caused by trauma, enzymatic digestion, surgery, or needles used during intervention [1]. Plastic stents have been rarely reported to cause visceral artery aneurysms by constantly irritating and breaching the vessel wall [2]. In our patient, a combination of enzymatic digestion and the presence of a transmural stent possibly led to a breach in the GDA wall because the tip of the stent was found closely abutting the abnormal segment of the GDA.

Endoscopy_UCTN_Code_CPL_1AL_2AD

Competing interests: None

\section{Surinder Singh Rana', Puneet Chha- bra', Chirag Ahuja², Deepak Gunjan', Deepak Kumar Bhasin ${ }^{1}$}

${ }^{1}$ Department of Gastroenterology, Post Graduate Institute of Medical Education and Research (PGIMER), Chandigarh, India

2 Department of Radiology, Post Graduate Institute of Medical Education and Research (PGIMER), Chandigarh, India

\section{References}

1 Young R, Gagandeep S, Grant E et al. Gastroduodenal artery pseudoaneurysm secondary to pancreatic head biopsy. J Ultrasound Med 2004; 23: 997-1001

2 Park JY, Ryu H, Bang $S$ et al. Hepatic artery pseudoaneurysm associated with plastic biliary stent. Yonsei Med J 2007; 48: 546 548
Bibliography

Dol http://dx.doi.org/

10.1055/s-0034-1393589

Endoscopy 2015; 47: E631-E632

(c) Georg Thieme Verlag KG

Stuttgart · New York

ISSN 0013-726X

Corresponding author Surinder Singh Rana, MD

Department of Gastroenterology PGIMER

Chandigarh - 160012

India

Fax: +91-172-2744401

drsurinderrana@yahoo.co.in 